\title{
Herramienta clínica para la búsqueda de biomarcadores de enfermedades neurológicas
}

\author{
Natalia Flórez \\ PhD Ingeniería Electrónica, \\ Universitat Politècnica de València \\ Investigador departamento de Radiología \\ Hospital Quirón , Valencia, España \\ Coordinadora Grupo UNITEL, \\ Universidad Santo Tomás de Aquino \\ Bucaramanga, Colombia \\ nataliaf@mail.ustabuca.edu.co
}

\author{
David Moratal \\ PhD Ingeniería Electrónica \\ Universitat Politècnica de València \\ Docente e investigador del \\ Departamento de Ingeniería Electrónica \\ Centro de Biomateriales e Ingeniería Tisular \\ Universitat Politècnica de València \\ Valencia, España \\ dmoratal@eln.upv.es·http://dmoratal.webs.upv.es
}

\author{
Luis Martí-Bonmatí \\ PhD Medicina y Cirugía \\ Universidad de Valencia \\ Jefe Departamento de Radiología \\ Hospital Quirón de Valencia \\ Valencia, España \\ marti_lui@gva.es
}

\begin{abstract}
Resumen- El solapamiento que presentan algunas enfermedades neurológicas en la clínica justifica la necesidad de establecer biomarcadores que sirvan como apoyo en el diagnóstico y seguimiento de la enfermedad. El análisis de los fluidos intracraneales (sangre y líquido cefalorraquídeo) juega un rol importante en la búsqueda de estos biomarcadores. Las secuencias dinámicas de resonancia magnética en contraste de fase (RM-CF) con sincronismo cardíaco permiten cuantificar el flujo de líquido cefalorraquídeo (LCR) y de la sangre durante un ciclo cardíaco. No obstante, la medición de flujo mediante secuencias de imágenes de RM-CF (IRM-CF) es precisa y reproducible siempre que se use un protocolo de adquisición y técnicas de posproceso adecuadas que permitan segmentar las regiones de interés (ROI) independientemente del operador y admitan corregir los errores introducidos por diversos artefactos. El presente trabajo describe una herramienta software de fácil manejo para el procesamiento de IRM-CF. Esta herramienta incluye métodos de segmentación, corrección de artefactos, cálculo de parámetros de flujo y módulos para la gestión de resultados y estudios estadísticos. La herramienta fue empleada por un radiólogo especializado para cuantificar el LCR en el acueducto de Silvio y nivel perimedular C2C3, flujo arterial en las arterias carótidas y vertebrales y flujo venoso en el seno sagital recto y superior en dos pacientes, uno con hidrocefalia normopresiva y el otro con una Malformación de Chiari. Los resultados obtenidos son comparados con parámetros de normalidad de flujo obtenidos previamente en 14 sujetos sanos. La herramienta brinda un gran número de opciones que permiten al usuario segmentar de una manera más práctica la región que se va a analizar, corregir artefactos y cuantificar parámetros, sirviendo como apoyo para el establecimiento de biomarcadores de la enfermedad.
\end{abstract}

Palabras clave- Hidrocefalia, líquido cefalorraquídeo posproceso, resonancia magnética, sangre.

\begin{abstract}
The comorbidity of diverse neurological disorders is one of the major problems at the moment to carry out a correct diagnosis. Therefore it is necessary to establish different biomarkers that facilitate the diagnostic and disease monitoring. The analysis of intracranial fluids (blood and CSF) plays an important role in the search for these biomarkers. The cine sequences of phase contrast magnetic resonance imaging (PC-MRI) with cardiac synchronism allow to quantify the CSF and blood flows during a cardiac cycle. However, flow measurement with PC-MRI is accurate and reproducible if an adequate acquisition protocol is used. The reproducibility and accuracy of the measures also depend on the use of adequate post-processing techniques that allow to segment the regions of interest (ROI), with great consistency and independency of the operator and admit to correct the errors introduced by different artifacts. The current work describes a friendly image post-processing tool that serves of support to carry out quantitative analysis of the flow dynamics in the main vessels and CSF spaces. This tool integrates different segmentation and correction methods, calculation of flow parameters and modules for the management of results and statistical studies. The tool was used by a radiologist in two patients (with normal pressure hydrocephalus and Chiari malformation) to quantify the CSF at the aqueduct of Sylvius and C2C3 level, blood flow in the carotid and vertebral arteries and venous flow in the straight and sagittal superior sinus. The results are compared with normal flow parameters obtained previously in 14 healthy subjects. The tool provides a number of options that allow the user to segment in a quick way the regions to analyze, to correct artifacts and to quantify parameters as aid for the establishment of disease biomarkers.
\end{abstract}

Key words- Hydrocephalus, cerebrospinal fluid, post-processing, magnetic resonance, blood. 


\section{INTRODUCCIÓN}

Las enfermedades neurológicas son trastornos que afectan el sistema nervioso central (SNC). La comorbidad o solapamiento que presentan en la clínica algunas de estas enfermedades dificulta su diagnóstico [1-6]. Diversos estudios han asociado enfermedades neurológicas y cerebrovasculares a desequilibrios en la dinámica de los fluidos intracraneales [7-10]. Las secuencias de resonancia magnética en contraste de fase (RM-CF) han abierto nuevas vías para el estudio de los cambios fisiológicos sobre la dinámica de fluidos ocasionada por diversos disturbios neurológicos, lo que facilita, establecer biomarcadores que incrementan la fiabilidad en el diagnóstico.

La RM-CF permite cuantificar la dinámica de fluidos dentro de las principales arterias, venas y espacios de líquido cefalorraquídeo (LCR) durante el ciclo cardíaco cuando se utiliza junto con algoritmos de sincronización cardiaca [11-13]. Desafortunadamente, la cuantificación de flujo a través de RM-CF está sujeta a diferentes fuentes de variabilidad e inexactitudes. La segmentación manual de la región que se va a analizar es una de las principales fuentes de variabilidad. El efecto de volumen parcial, la dispersión de fase intravóxel, el submuestreo (aliasing) y los errores residuales sistemáticos [14,15] son las principales fuentes de inexactitud. Estos factores justifican la necesidad de utilizar protocolos de adquisición adecuados y de emplear metodologías adecuadas de posproceso que permitan analizar la dinámica de los líquidos intracraneales de manera fiable y reproducible, y así obtener parámetros de referencia que sirvan de ayuda para el diagnóstico y seguimiento de diferentes enfermedades neurológicas.

Los principales fabricantes de equipos de RM proveen de software para analizar la dinámica de los fluidos con funciones limitadas. Algunos de ellos permiten definir regiones de interés (region of interest, ROI) circulares o elípticas de manera manual o semiautomática, tedioso para pequeños vasos o regiones complejas.

El presente trabajo describe una herramienta software de fácil manejo para el procesamiento de IRM-CF. Esta herramienta incluye métodos de segmentación, de corrección de artefactos, de cálculo de parámetros de flujo y módulos para la gestión de resultados y estudios estadísticos.

La herramienta fue empleada por un radiólogo especializado para cuantificar en dos pacientes: uno con hidrocefalia normopresiva (hombre de 50 años) y otro con Malformación de Chiari (hombre de 55 años) el LCR en el acueducto de Silvio y nivel perimedular $\mathrm{C} 2 \mathrm{C} 3$, flujo arterial en las carótidas y vertebrales y flujo venoso en el seno sagital recto y superior. Los resultados obtenidos son comparados con parámetros de normalidad de flujo obtenidos previamente en 14 sujetos sanos [16].

\section{MATERIALES Y MÉTODOS}

\section{A. Entorno de trabajo}

La herramienta que se presenta se desarrolló en colaboración constante entre personal médico e ingenieros especializados. Se utilizó para todo el proceso el paquete informático MATLAB R7 (The MathWorks, Inc., Natick, MA, USA), y todas las mediciones se realizaron "off line" en una estación de trabajo (HP Workstation, Intel Core $2.24 \mathrm{GHz}$, 2Gb RAM).

\section{B. Adquisición de las imágenes}

Las secuencias de imágenes se realizaron en el Servicio de Radiología del Hospital Quirón de Valencia (España) con un equipo de RM de 3 Teslas (Achieva Intera, Philips Medical Systems, Best, Países Bajos).

Para cada estudio se realizarán cuatro adquisiciones. La primera secuencia perpendicular al acueducto de Silvio, con velocidad de codificación (velocity encoding, $\mathrm{V}_{\text {enc }}$ ) de $15 \mathrm{~cm} / \mathrm{s}$; la segunda paralela al nivel C2C3 para el cálculo de LCR perimedular, con $V_{\text {enc }}$ de $7 \mathrm{~cm} / \mathrm{s}$; una tercera secuencia también paralela al nivel C2C3 para cálculo de flujo arterial (carótidas internas y vertebrales) con $V_{\text {enc }}$ de $100 \mathrm{~cm} / \mathrm{s}$ y una cuarta secuencia perpendicular al seno sagital superior y recto para analizar el flujo venosos con $V_{\text {enc }}$ de $80 \mathrm{~cm} / \mathrm{s}$ (Fig. 1) Los parámetros más relevantes de la secuencia en contraste de fase son el tamaño de la matriz = 512 x 512, un campo de visión de $170 \mathrm{~mm}$, lo que genera un tamaño final del voxel adquirido de 0,33 x 0,33 x $5 \mathrm{~mm}$, número de señales promediadas $(N S A)=2$, ángulo de excitación $=15^{\circ}$ y 25 
puntos temporales o muestras por ciclo cardiaco adquiridos mediante sincronización cardiaca retrospectiva durante todo el ciclo cardiaco; tiempo de eco y tiempo de repetición mínimos; velocidad de codificación $\left(\mathrm{V}_{\text {enc }}\right)$ de $15 \mathrm{~cm} / \mathrm{s}$ para LCR en el acueducto de Silvio, de $7 \mathrm{~cm} / \mathrm{s}$ para LCR perimedular, $100 \mathrm{~cm} / \mathrm{s}$ para flujo arterial y $80 \mathrm{~cm} / \mathrm{s}$ para flujo arterial.

El posproceso de las imágenes se inicia marcando un punto semilla dentro de la región analizada es ésta la única interacción del usuario en el proceso. Para la segmentación de las regiones de interés (regions of interest, ROI), se utiliza la información temporal del flujo a lo largo del ciclo cardiaco, de esta manera el método es independiente de la relación intensidad-señal, inconveniente que se presenta en los métodos que usan la información espacial para realizar la segmentación.

FIG. 1. CORTE SAGITAL DE UNA SECUENCIA DE RM PULSO DE INVERSIÓN PARA LA LOCALIZACIÓN DE LA ADQUISICIÓN DE LAS IMÁGENES PERPENDICULAR AL 1) ACUEDUCTO DE SILVIO Y SENOS VENOSOS Y 2) NIVEL PERIMEDULAR C2C3

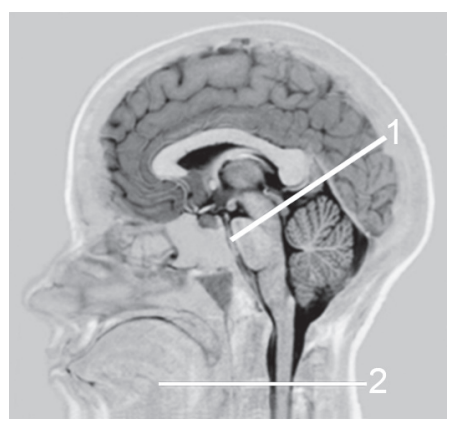

Fuente: Los autores

Segmentación de la región de interés y corrección de artefactos

Para analizar la dinámica de los fluidos intracraneales (sangre y LCR) se implementaron dos métodos dinámicos de segmentación basados en el comportamiento del flujo. El primer método permite la segmentación de flujo en regiones con perfiles de velocidad parabólico o plano como el acueducto de Silvio y vasos vasculares mediante técnicas de umbralización [17]. El segundo método permite segmentar regiones con anatomía compleja y perfiles de flujo híperdinámico mediante correlaciones, umbralizaciones y técnicas no supervisadas de clasificación k-medias [18].

Para corregir los errores residuales causados por la supresión imperfecta de las corrientes inducidas y la contribución a la señal de los pequeños movimientos que presenta el mesencéfalo por la transmisión del pulso vascular, se calcula un valor promedio del desplazamiento (offset) en regiones donde el movimiento es mínimo o ausente durante el ciclo cardiaco (el parénquima mesencefálico) para posteriormente sustraerlo de las medidas dinámicas obtenidas [17].

Dado que el valor seleccionado de Venc influye en la presencia de submuestreo en la secuencia de imágenes y dificulta las medidas de flujo, el valor de Venc debe ser mayor pero próximo a la máxima velocidad de flujo esperada para maximizar la relación señal a ruido y obtener medidas más precisas [19]. La existencia de píxeles con aliasing se detecta y corrige automáticamente con el método descrito previamente $[17,18]$.

En la Fig. 2 se observa la ventana principal del software donde están incluidos los diferentes métodos de segmentación y corrección de artefactos; también se incluyen diversas funciones para la manipulación y visualización de las imágenes.

\section{Cuantificación de parámetros de flujo}

Una vez son segmentadas las ROls se calculan diversos parámetros de flujo:

- Flujo promedio (Mean flow) [ml/min]: calculado como la media del valor absoluto de las medidas en sístole y diástole.

- Volumen sistólico (Systolic stroke volume) [ $\mu \mathrm{l}]$ : Volumen de flujo que se incrementa en sístole por encima del volumen promedio, calculado como el área bajo la curva en sístole.

- Tiempos (Time) [\% cc]: Porcentaje de ciclo cardíaco medido entre picos sistólicos. Se toma como referencia el pico sistólico de la entrada arterial en el $0 \%$.

- Índice de complianza (Compliance index): Da una estimación de la complianza arterio-cerebral. Es analizada respecto al incremento de volumen arterial en sístole. Se obtiene al substraer el volumen sistólico en el acueducto del volumen sistólico arterial y dividiendo este valor por el volumen sistólico del acueducto.

- Índice de pulsatilidad (Pulsatibility index): Relación entre la diferencia del flujo máximo sistólico y diastólico frente a la media de flujo [20].

- Amplitud del gradiente de presión (Pressure gradient) [mmHg /cm]: Cambios de presión 
durante un ciclo cardíaco son derivados de cambios de velocidad de flujo mediante las ecuaciones de Navier Stokes para fluidos incomprensibles (flujos con densidad constante) en un tubo rígido. Las ecuaciones de Navier Stokes describen cómo la velocidad, presión, temperatura y densidad de un flujo están relacionadas.

- Relación de volumen por ciclo (Stroke volume ratio) [\%]: Porcentaje de volumen desplazado durante un ciclo cardíaco desde el sistema ventricular al espacio subaracnoideo. El volumen por ciclo (VC) es calculado como el promedio del volumen desplazado en sístole y diástole.

- Producción de LCR supratentorial (CSF production) $[\mathrm{mL} / \mathrm{min}]$ : Diferencia entre el volumen de flujo sistólico y el diastólico por unidad de tiempo.

FIG 2. VENTANA PRINCIPAL DEL SOFTWARE

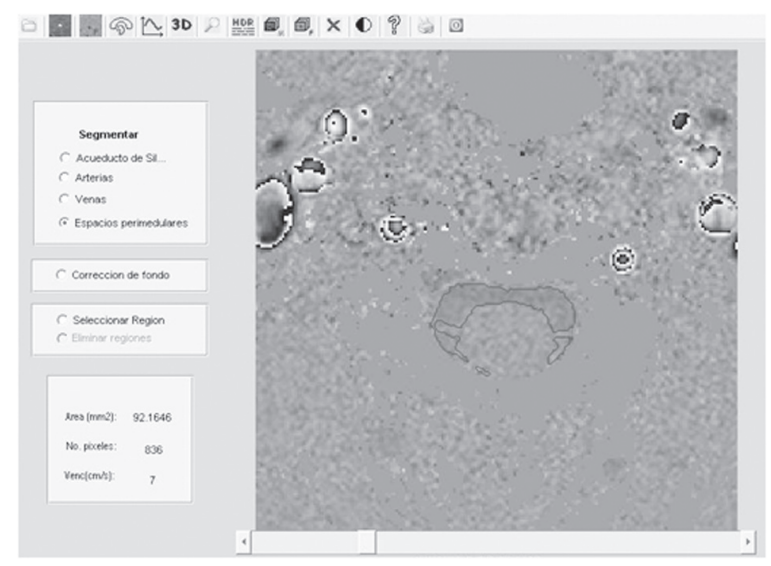

Fuente: Los autores

\section{Gestíón de resultados}

Los diferentes parámetros cuantificados en cada sujeto son almacenados en una base de datos y, a su vez, exportados a archivos .xls y .sav para estudios estadísticos y en .pdf para adjuntar al informe radiológico.

\section{RESULTADOS}

En el paciente con malformación de Chiari I la dinámica intracraneal revela desequilibrios en la entrada de flujo arterial, salida de flujo venoso por el seno recto y desplazamiento de LCR en el C2C3. La relación de volumen por ciclo fue incrementada en comparación con los sujetos sanos (Tabla 1).
Mientras que en el paciente con hidrocefalia normopresiva, hay un flujo hiperpulsátil en el acueducto de Silvio, una disminución de la entrada de flujo arterial y del flujo venoso por el seno recto. La relación de volumen por ciclo fue incrementada en comparación con los sujetos sanos (Tabla 2).

La Fig. 3 reflejan pérdida de complianza cerebral mediante la distribución temporal representada en porcentaje de ciclo cardíaco.

FIG. 3. DISTRIBUCIÓN TEMPORAL DE LOS FLUIDOS INTRACRANEALES. ST: SENO RECTO, SSS: SENO SAGITAL SUPERIOR, AS: ACUEDUCTO DE SILVIO, C2C3: NIVEL PERIMEDULAR
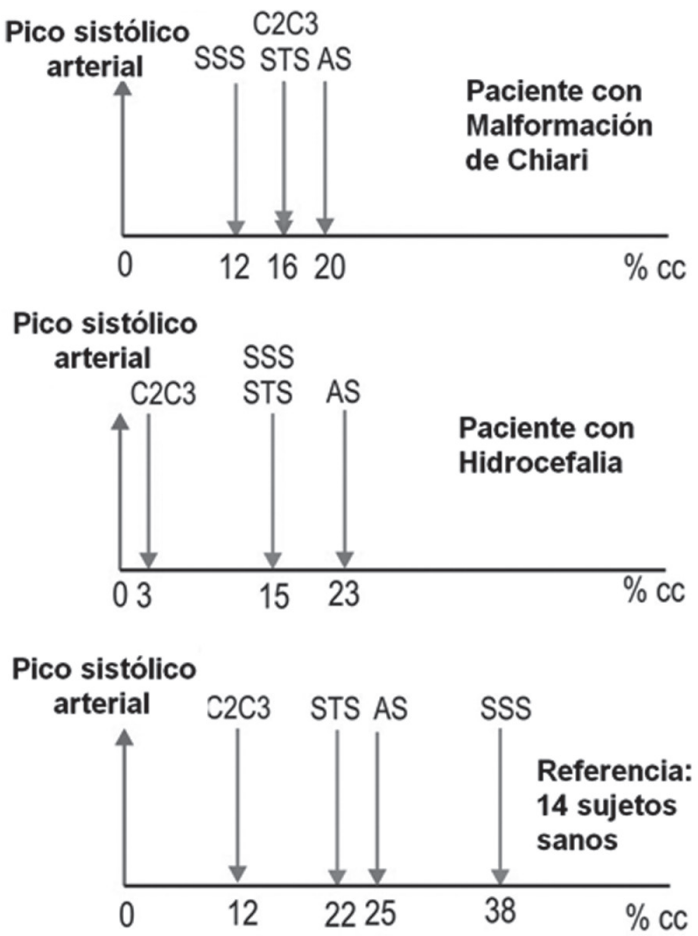

Fuente: Los autores

TABLA 1. PARÁMETROS CUANTIFICADOS EN UN PACIENTE CON MALFORMACIÓN DE CHIARI

\begin{tabular}{|c|c|c|c|c|c|c|}
\hline \multicolumn{2}{|l|}{ Parámetros } & $\begin{array}{l}\text { Entrada } \\
\text { arterial }\end{array}$ & $\begin{array}{l}\text { Acueducto } \\
\text { de Silvio }\end{array}$ & $\mathrm{C} 2 \mathrm{C} 3$ & Seno recto & $\begin{array}{l}\text { Seno } \\
\text { superior }\end{array}$ \\
\hline \multicolumn{2}{|c|}{ Flujo promedio (ml/min) } & $\begin{array}{c}369 \\
660 \pm 104\end{array}$ & $\begin{array}{c}6 \\
5 \pm 2\end{array}$ & $\begin{array}{c}34 \\
75 \pm 12\end{array}$ & $\begin{array}{c}99 \\
90 \pm 10\end{array}$ & $\begin{array}{c}289 \\
320 \pm 90\end{array}$ \\
\hline \multicolumn{2}{|c|}{ Volumen sistólico ( $\mu \mathrm{l} /$ /ciclo) } & $\begin{array}{c}778 \\
1300 \pm 196\end{array}$ & $\begin{array}{c}40 \\
34 \pm 18\end{array}$ & $\begin{array}{c}221 \\
544 \pm 122\end{array}$ & $\begin{array}{c}120 \\
58 \pm 18\end{array}$ & $\begin{array}{c}279 \\
247 \pm 76\end{array}$ \\
\hline \multicolumn{2}{|c|}{ Retrasos (\% ciclo cardiaco) } & & $\begin{array}{c}20 \\
25 \pm 13\end{array}$ & $\begin{array}{c}16 \\
12 \pm 10\end{array}$ & $\begin{array}{c}16 \\
22 \pm 4\end{array}$ & $\begin{array}{c}12 \\
38 \pm 16\end{array}$ \\
\hline \multicolumn{2}{|l|}{ Índice de complianza } & & $\begin{array}{c}18 \\
35 \pm 10\end{array}$ & $\begin{array}{c}2.5 \\
1.10 \pm 0.45\end{array}$ & $\begin{array}{l}21.8 \\
21 \pm 6\end{array}$ & $\begin{array}{c}1.8 \\
5.20 \pm 3\end{array}$ \\
\hline \multicolumn{2}{|l|}{ Índice de pulsatibilidad } & $\begin{array}{c}1.23 \\
1.03 \pm 0.15\end{array}$ & $\begin{array}{c}0.62 \\
0.35 \pm 0.26\end{array}$ & $\begin{array}{c}0.15 \\
1.42 \pm 0.33\end{array}$ & $\begin{array}{c}0.58 \\
0.33 \pm 0.04\end{array}$ & $\begin{array}{c}0.42 \\
0.39 \pm 0.10\end{array}$ \\
\hline \multicolumn{2}{|c|}{$\begin{array}{l}\text { Amplitud de gradiente de pre- } \\
\text { sión (mmHg) }\end{array}$} & $\begin{array}{c}0.16 \\
0.31 \pm 0.08\end{array}$ & $\begin{array}{c}0.07 \\
0.07 \pm 0.03\end{array}$ & $\begin{array}{c}0.05 \\
0.07 \pm 0.02\end{array}$ & $\begin{array}{c}0.09 \\
0.11 \pm 0.03\end{array}$ & $\begin{array}{c}0.08 \\
0.09 \pm 0.03\end{array}$ \\
\hline $\begin{array}{l}\text { Relación de volumen } \\
\text { por ciclo (\%) }\end{array}$ & \multicolumn{2}{|c|}{$\begin{array}{c}18 \\
5.26 \pm 2.20\end{array}$} & & & & \\
\hline $\begin{array}{l}\text { Producción de LCR } \\
\text { (ml/min) }\end{array}$ & \multicolumn{2}{|c|}{$\begin{array}{c}-0.41 \\
-0.24 \pm 0.19\end{array}$} & & & & \\
\hline
\end{tabular}


TABLA 2. PARÁMETROS CUANTIFICADOS EN UN PACIENTE CON HIDROCEFALIA NORMOPRESIVA

\begin{tabular}{|c|c|c|c|c|c|}
\hline Parámetros & $\begin{array}{l}\text { Entrada } \\
\text { arterial }\end{array}$ & $\begin{array}{l}\text { Acueducto } \\
\text { de Silvio }\end{array}$ & $\mathrm{C} 2 \mathrm{C} 3$ & Seno recto & $\begin{array}{l}\text { Seno } \\
\text { superior }\end{array}$ \\
\hline Flujo promedio (ml/min) & $\begin{array}{c}369 \\
660 \pm 104\end{array}$ & $\begin{array}{c}17 \\
5 \pm 2\end{array}$ & $\begin{array}{c}81 \\
75 \pm 12\end{array}$ & $\begin{array}{c}54 \\
90 \pm 10\end{array}$ & $\begin{array}{c}220 \\
320 \pm 90\end{array}$ \\
\hline Volumen sistólico ( $\mu \mathrm{l} / \mathrm{ciclo})$ & $\begin{array}{c}1100 \\
1300 \pm 196\end{array}$ & $\begin{array}{c}117 \\
34 \pm 18\end{array}$ & $\begin{array}{c}559 \\
544 \pm 122\end{array}$ & $\begin{array}{c}59 \\
58 \pm 18\end{array}$ & $\begin{array}{c}300 \\
247 \pm 76\end{array}$ \\
\hline Retrasos (\% ciclo cardiaco) & & $\begin{array}{c}23 \\
25 \pm 13\end{array}$ & $\begin{array}{c}3 \\
12 \pm 10\end{array}$ & $\begin{array}{c}15 \\
22 \pm 4\end{array}$ & $\begin{array}{c}15 \\
38 \pm 16\end{array}$ \\
\hline Índice de complianza & & $\begin{array}{c}8 \\
35 \pm 10\end{array}$ & $\begin{array}{c}0.95 \\
1.10 \pm 0.45\end{array}$ & $\begin{array}{c}17 \\
21 \pm 6\end{array}$ & $\begin{array}{c}2.7 \\
5.20 \pm 3\end{array}$ \\
\hline Índice de pulsatibilidad & $\begin{array}{c}1.16 \\
1.03 \pm 0.15\end{array}$ & $\begin{array}{c}0.1 \\
0.35 \pm 0.26\end{array}$ & $\begin{array}{c}0.6 \\
1.42 \pm 0.33\end{array}$ & $\begin{array}{c}0.48 \\
0.33 \pm 0.04\end{array}$ & $\begin{array}{c}0.58 \\
0.39 \pm 0.10\end{array}$ \\
\hline $\begin{array}{l}\text { Amplitud de gradiente de pre- } \\
\text { sión (mmHg) }\end{array}$ & $\begin{array}{c}0.23 \\
0.31 \pm 0.08\end{array}$ & $\begin{array}{c}0.07 \\
0.07 \pm 0.03\end{array}$ & $\begin{array}{c}0.03 \\
0.07 \pm 0.02\end{array}$ & $\begin{array}{c}0.13 \\
0.11 \pm 0.03\end{array}$ & $\begin{array}{c}0.11 \\
0.09 \pm 0.03\end{array}$ \\
\hline
\end{tabular}

\begin{tabular}{|l|c|}
\hline $\begin{array}{l}\text { Relación de volumen } \\
\text { por ciclo (\%) }\end{array}$ & $\begin{array}{c}20 \\
5.26 \pm 2.20\end{array}$ \\
\hline $\begin{array}{l}\text { Producción de LCR } \\
\text { (ml/min) }\end{array}$ & $\begin{array}{c}-0.39 \\
-0.24 \pm 0.19\end{array}$ \\
\hline
\end{tabular}

Fuente: Los autores

\section{CONCLUSIONES}

Los resultados preliminares obtenidos justifican la necesidad de establecer parámetros de referencia en diferentes grupos de patologías. Las técnicas de posproceso de imagen incluidas en la herramienta desarrollada servirán como apoyo a la investigación clínica de enfermedades neurológicas.

El software presentado en este trabajo brinda un gran número de opciones que permiten al usuario segmentar de una manera más práctica la región a analizar, corregir artefactos y cuantificar parámetros relacionados con la dinámica de flujo cerebral.

\section{AGRADECIMIENTOS}

Este trabajo ha sido financiado parcialmente por una beca de investigación de la Fundación del Colegio de Médicos de Valencia (España). La herramienta presentada para el análisis cuantitativo de flujo usada en este trabajo está registrada en el Centro de Transferencia Tecnológica de la Universidad Politécnica de Valencia con el número de registro CTT-007804.

\section{REFERENCIAS}

[1] G. A. Bateman, C. R. Levi, P. Schofield, Y. Wang, and E. C. Lovett, "The pathophysiology of the aqueduct stroke volume in normal pressure hydrocephalus: can co-morbidity with other forms of dementia be excluded?," Neuroradiology, vol. 47, no. 10, pp. 741-748, Jul. 2005.
[2] S. Savolainen, L. Paljarvi, and M. Vapalahti, "Prevalence of Alzheimer's disease in patients investigated for presumed normal pressure hydrocephalus: a clinical and neuropathological study," Acta Neurochir. (Wien), vol. 141, no. 8, pp. 849-853, 1999

[3] G. A. Bateman, "Pulse wave encephalopathy: a spectrum hypothesis incorporating Alzheimer's disease, vascular dementia and normal pressure hydrocephaIus," Med Hypotheses, vol. 62, no. 2, pp. 182-187, 2004.

[4] G. A. Bateman, "The pathophysiology of idiopathic normal pressure hydrocephalus: cerebral ischemia or altered venous hemodynamics?," AJNR Am. J. Neuroradiol., vol. 47 , no. 10 , pp. $741-748, .2005$

[5] W. G. Bradley, Jr., A. R. Whittemore, A. S. Watanabe, S. J. Davis, L. M. Teresi, and M. Homyak, "Association of deep white matter infarction with chronic communicating hydrocephalus: implications regarding the possible origin of normal pressure hydrocephalus," AJNR Am J Neuroradiol, vol. 12, no. 1, pp. 31-39, 1991.

[6] G. A. Bateman, "Pulse wave encephalopathy: a comparative study of the hydrodynamics of leukoaraiosis and normal pressure hydrocephalus," Neuroradiology, vol. 44, no. 9, pp. 740-748, 2002.

[7] V. M. Haughton, F. R. Korosec, J. E. Medow, M. T. Dolar, and B. J. Iskandar, "Peak systolic and diastolic CSF velocity in the foramen magnum in adult patients with Chiari I malformations and in normal control participants," AJNR Am J Neuroradiol, vol. 24, no. 2, pp. 169176, 2003.

[8] R. A. Armonda, C. M. Citrin, K. T. Foley, and R. G. Ellenbogen, "Quantitative cine-mode magnetic resonance imaging of Chiari I malformations: an analysis of cerebrospinal fluid dynamics," Neurosurgery, vol. 35, no. 2, pp. 214-223, 1994.

[9] O. Baledent, C. Gondry-Jouet, M. E. Meyer, G. de Marco, D. Le Gars, M. C. Henry-Feugeas, and I. Idy-Peretti, "Relationship between cerebrospinal fluid and blood dynamics in healthy volunteers and patients with communicating hydrocephalus," Invest Radiol, vol. 39, no. 1, pp. 45-55, 2004.

[10] D. Greitz, T. Greitz, and T. Hindmarsh, "A new view on the CSF-circulation with the potential for pharmacological treatment of childhood hydrocephalus," Acta Paediatr, vol. 86, no. 2, pp. 125-132, 1997.

[11] O. Baledent, C. Gondry-Jouet, S. Stoquart-Elsankari, R. Bouzerar, D. Le Gars, and M. E. Meyer, "Value of phase contrast magnetic resonance imaging for investigation of cerebral hydrodynamics," J Neuroradiol, vol. 33, no. 5, pp. 292-303, 2006.

[12] W. G Bradley, K. E Kortman, B. Burgoyne. "Flowing Cerebrospinal-Fluid in Normal and Hydrocephalic States 
- Appearance on MR Images," Radiology, vol. 159, no. 3, pp. 611-616, 1986.

[13] W.G Bradley, "Magnetic Resonance Imaging in the Evaluation of Cerebrospinal Fluid Flow Abnormalities." Magnetic Resonance Quarterly, vol. 8, no. 3, pp. 169196, 1992.

[14] R. L. Wolf, R. L. Ehman, S. J. Riederer, and P. J. Rossman, "Analysis of systematic and random error in MR volumetric flow measurements," Magn Reson Med, vol. 30, no. 1, pp. 82-91, 1993.

[15] G. Greil, T. Geva, S. E. Maier, and A. J. Powell, "Effect of acquisition parameters on the accuracy of velocity encoded cine magnetic resonance imaging blood flow measurements," J Magn Reson Imaging, vol. 15, no. 1, pp. 47-54, 2002.

[16] N. Flórez, L Martí-Bonmatí, J. Forner, E. Arana, D. Moratal, "Estimación de la dinámica de líquidos intracraneales mediante análisis cuantitativo de imágenes de resonancia magnética de contraste de fase". Radiologia, 2009 Oct 22. [Epub ahead of print]

[17] Y. N. Flórez, D. Moratal, J. Forner, L. Marti-Bonmati, E. Arana, U. Guajardo-Hernandez, and J. Millet-Roig, "Semiautomatic analysis of phase contrast magnetic resonance imaging of cerebrospinal fluid flow through the aqueduct of Sylvius," Magn Reson Mater Phy, vol. 19, no. 2, pp. 78-87, 2006.

[18] Flórez N, “Intracranial Fluids Dynamics: A Quantitative Evaluation by Means of Phase-Contrast Magnetic Resonance Imaging", Tesis Doctoral, Universidad Politècnica de Valencia (España), 2009

[19] N. J. Pelc, M. A. Bernstein, A. Shimakawa, and G. H. Glover, "Encoding strategies for three-direction phasecontrast MR imaging of flow," J Magn Reson Imaging, vol. 1, no. 4, pp. 405-413, 1991.

[20] R. G. Gosling and D. H. King, "Arterial assessment by Doppler-shift ultrasound," Proc. R. Soc. Med, vol. 67, no. 6 Pt 1, pp. 447-449, 1974. 